$10 / 09 / 02$

\title{
FLOW PATTERNS DURING FRICTION STIR WELDING
}

M. Guerra, C. Schmidt, J.C. McClure, L.E. Murr, A.C. Nunes*
Metallurgical and Materials Engineering Department

University of Texas El Paso

*NASA, Marshall Space Flight Center, Huntsville, AL

\section{ABSTRACT}

Friction Stir Welding is a relatively new technique for welding that uses a cylindrical pin or nib inserted along the weld seam. The nib (usually threaded) and the shoulder in which it is mounted are rapidly rotated and advanced along the seam. Extreme deformation takes place leaving a fine equiaxed structure in the weld region. The flow of metal during Friction Stir Welding is investigated using a faying surface tracer and a nib frozen in place during welding. It is shown that material is transported by two processes. The first is a wiping of material from the advancing front side of the nib onto a zone of material that rotates and advances with the nib. The material undergoes a helical motion within the rotational zone that both rotates and advances and descends in the wash of the threads on the nib and rises on the outer part of the rotational zone. After one or more rotations, this material is sloughed off in its wake of the nib, primarily on the advancing side. The second process is an entrainment of material from the front retreating side of the nib that fills in between the sloughed off pieces from the advancing side. 


\section{INTRODUCTION}

Friction Stir Welding $\left.{ }^{1}\right]$ has been put into use very rapidly by a usually conservative welding community and certainly must be considered to be one of the major advances in welding technology in recent years. The weld is made by inserting a usually threaded pin or nib into the faying surface of butt welded parts. The nib is typically slightly shorter than the thickness of the work piece and its diameter is approximately the thickness of the work piece. The nib is mounted in a shoulder that may be three times the diameter of the nib. The nib and shoulder are pressed against the work piece and rotated at several hundred revolutions per minute and advanced along the faying surface.

Friction Stir Welding has been primarily used on aluminum alloys and produces nearly defect free welds for such demanding applications as space hardware at lower cost than conventional fusion welds.

Like any new technology many unanswered questions remain, but even such a fundamental question as how material flows from front to back around the nib remains the subject of various investigations. For aluminum welds, temperatures near the rotating nib never reach the melting point $\left[{ }^{2,3}\right]$ but quite complicated flow (Fig.1) has been observed in welds made on bimetals such as $\mathrm{Cu} / \mathrm{Al}\left[{ }^{4}\right]$ in which vortices of $\mathrm{Cu}$ penetrating into $\mathrm{Al}$ can be readily seen. Vertical flow as well as rotational flow of material around the nib must also be important since the FSW nib is usually threaded and turned in such a direction that material is pushed down by the threads (for example, a right hand thread turning counterclockwise). Reversing the direction of rotation of the nib (without changing the hand of the threads) generally results in an inferior weld. 
Several recent studies on material flow have been published. Before discussing these papers, a convention is needed to distinguish between the two sides of a FSW weld. In this paper it will be assumed that the relative motion between the tool and the workpiece is due to a moving tool and a stationary workpiece. Furthermore, the sides of the friction stir weld will be referred to as the "advancing side" when the rotational motion of the nib and its translation motion are in the same direction. The "retreating side" is the side in which the rotational motion is in the opposite direction to that of the translational motion.

Colligan $^{5}$ followed material flow in 6061 and 7075 aluminum by imbedding small steel balls as tracers into groves cut into the workpiece parallel to the weld direction. Grooves were cut parallel to the weld direction but at various distances from the weld center line and at various depths. After welding, the distribution of steel balls was revealed by radiography in both the plan view and a cross sectional view. Results are displayed nicely in the original paper but in general, the work showed that material striking the pin on the advancing side of the weld will be swept around the rotating nib often deposits on the retreating side behind the nib. Material striking the nib on the retreating side usually (depending on its depth) will also be deposited on the retreating side behind the weld. Cross sectional radiographs indicate for most positions a lifting of the markers to points nearer the shoulder of the tool. Vertical motion of the markers is not large and most material stays at roughly the same depth into the sample at which it started. Colligan supplements this study with "stop action" data in which the threaded nib is permitted to unwind its way out of the workpiece very rapidly leaving behind a record of the material that was against the nib. Polished sections through this material were 
tentatively interpreted to indicate that some material was moved around the nib and deposited behind it. This is the "stirred" material from which the technique takes its name. The author points out that other material may have been squeezed or extruded around the nib on the retreating side. Colligan thus interprets the friction stir welding process as being due to both stirring and to extrusion of material.

In another recent work, $\left[{ }^{6}\right]$ Reynolds used a different marker configuration. He placed thin sheets of 5454 Aluminum into slots milled into the faying surfaces of his 2195 workpiece. The slots were milled near the bottom, in the middle, and near the top of the workpiece and extended from the weld center line to beyond the thermomechanical zone at the edge of the weld. After the weld was completed, the workpiece was thinned by mechanical milling in steps and after each step the workpiece was polished and etched to reveal the position of the 5454 marker material.

The marker positions were digitized and combined into three dimensional maps indicating the flow. Only modest vertical flow was observed and the three dimensional maps could be interpreted as being the results of material extrusion from front to back around both sides of the nib. In this model, the role of the rotating nib is to provide frictional heating to make the extrusion possible.

Murr $\left.{ }^{7,8}\right]$ has shown that dynamic recrystalization plays a key role in material movement and has shown that for many similar examples of extreme deformation at very high strain rates solid state flow accommodates the process. These include shaped charges ${ }^{9}$, hypervelocity impact crater formation' $\left[{ }^{10,11}\right]$ and thick metal or alloy target penetration by ballistic energy rods $[12,13,14]$. 
Nunes $\left[{ }^{15}\right]$ has emphasized the importance of the rotational flow of material around the nib. He has synthesized a model of flow around the nib out of (1) a pure translation flow field, (2) a rigid disc rotation flow field (enclosing the nib), and (3) a radially symmetrical vortex ring flow field (inward at the shoulder, down at the nib threads, outward on the lower part of the nib, and upwards in the outer regions around the nib to complete the circulation. The rotating disc is bounded by a roughly cylindrical shear surface or rotational zone extending from slightly below the shoulder (where the flow is dominated by the shoulder rather than the threads) to near the bottom of the weld. Metal enters the rotational zone and is later sloughed off in the wake of the weld after one or more rotations. Continued forward motion results in the burial of incorporated metal under subsequently ingested layers. The purpose of this paper is to further develop the rotational model of material movement. It will be shown that both rotational motion or stirring and entrainment of material take place and combine to give the rather complicated structures found in the friction stir weld zone.

\section{EXPERIMENTAL PROCEDURE}

All welds were made on a Gorton Mastermill using a one piece nib and shoulder from $\mathrm{D} 2$ tool steel heat treated to $\mathrm{R}_{\mathrm{C}}=62$. The nib was $0.25 \mathrm{in} .(6.3 \mathrm{~mm})$ diameter and .23 in. $(5.8 \mathrm{~mm})$ long with standard $.25 / 20$ right hand threads and a $0.75 \mathrm{in.}$. (19mm) diameter shoulder machined perpendicular to the axis of the tool. The nib rotation direction was counterclockwise such that material was pushed downward by the threads. The bottom of the nib was ground flat with a set back from the back side of the sample of 
approximately .002 in. $(0.05 \mathrm{~mm})$. Rotation speeds were $1000 \mathrm{rpm}$ and translation velocity was $7.1 \mathrm{in} / \mathrm{minute}(3.0 \mathrm{~mm} / \mathrm{s})$. The nib had a $1^{\circ}$ lead angle.

Some welds were made on 0.25 inch $(6.3 \mathrm{~mm})$ thick 6061 Aluminum with a thin $0.0045 \mathrm{inch}(0.1 \mathrm{~mm})$ high purity $\mathrm{Cu}$ foil along the faying surface of the weld. After a stable weld had been established, the nib rotation and specimen translation were manually stopped as nearly simultaneously as possible to produce a nib "frozen" into the workpiece. The spindle was equipped with a mechanical brake and although it is difficult to estimate how quickly the welding operation stopped, it was nearly instantaneous because both the motor advancing the workpiece and the tool rotation motor are heavily loaded during welding. $\left[{ }^{16}\right]$ Plan view and transverse metallographic sections were made through the frozen nib as discussed below.

Other welds were made on a 0.25 inch $(6.3 \mathrm{~mm})$ thick workpiece in which the top half was 2195 aluminum and the bottom half was 6061 aluminum. When etched with Keller's reagent, these alloys appear very different and top to bottom flow can be seen easily.

Vickers's microhardness measurements were made in various regions of the welds.

\section{RESULTS AND DISCUSSION}

Fig. 2 shows a plan view made at midsection of the weld with the copper strip along the faying surface. The section was etched for 60 seconds with Keller's reagent. This long etching time is needed to bring out the dark features seen in Fig. 2. The direction of tool advance and rotation are indicated. The advancing side is on the right 
and the retreating side is on the left. Fig. 3 shows a transverse section through another frozen nib. In this figure, the nib is moving out of the page with the advancing side on the right and the retreating side on the left. Several interesting features can be seen in these figures.

The large circular feature near the center of Figure 2 is the nib seen in cross section. Surrounding the nib indicated by arrows $A$ is an open space with no material. A similar region was seen by Colligan $\left[{ }^{17}\right]$ in "stop action" sections taken when the nib was allowed to unwind from the work piece. Outside this open region (indicated by arrows B) is a nearly circular region (the "rotational zone") of dark heavily etched material. This region is approximately three times thicker than the distance that the nib moves in one revolution and is seen in the detail of Fig. 4 . It consists of fine grains which etched to produce facets that reflect light off the optic axis and hence appear dark. It will be shown that this zone rotates with the velocity of the nib.

Outside of the rotational zone is a "transition zone" marked by arrows $\mathrm{C}$. The zone is about twice as thick on the retreating side as it is on the advancing side. It is believed that material in this zone rotates, but its velocity decreases from the rotational velocity of the nib at the inner edge of the transition zone to zero at its outer edge. Ahead of the nib at the top of Figure 2 (Fig. 5 shows an enlarged view) the copper foil can be seen on edge. During specimen cutting some of the copper foil was dislodged and its space was filled with mounting compound but it is seen, however, that the foil curls around the nib at the outside of the rotational zone. A nominal strain can be calculated by noting that in one revolution of the nib, an amount of material ahead of the nib equal to $V / 2 \pi \omega$ ( $\omega$ and $V$ are the angular and linear velocity of the nib) is spread 
behind the nib over a distance equal to one half the circumference of the nib. This nominal strain is on the order of $10,000 \%$, and the foil is unable to sustain such very large elongations and tears apart into the small elongated particles seen light-colored in Fig. 5. These pieces are much smaller than other relevant dimensions in the welding process and provide good markers for material movement.

The copper particles seen in Fig. 5 start to curl around the rotational zone but shortly disappear from the plane of polish. It will later be shown that the threads on the nib create a secondary vortex or vertical flow of material within the rotational zone. This flow is upward at the outer edge of the rotational zone, inward at the top, downward with the wash of the threads, and finally outward at the bottom of the weld. On a typical plane of polish as seen in Fig. 2, the particles appear at various positions throughout the rotational zone depending on where on this complicated unwinding helical trajectory they came to rest when the weld was stopped.

The velocity of the vertical flow can be estimated from the equivalent vertical velocity of the threads. If three threads seen in Fig. 3 engage in the downward movement of metal it takes six revolutions for material to go down and then up within the rotational zone during which the nib travels $1.2 \mathrm{~mm}$ or about three times the radius of the rotational zone. Thus, any piece of material that enters the rotational zone, completes roughly one half of a round trip up and down in the vortex flow before it leaves the rotational zone. The large vertical motion of material during FSW can be appreciated in Fig. 6 which shows a cross section perpendicular to the weld direction made on a bimetallic specimen. The top half of the 0.25 in $(6.3 \mathrm{~mm})$ thick specimen was alloy 2195 and the bottom half of the specimen was alloy 6061 . The advancing side is on the right and the 
retreating side is on the left. Under Keller's reagent (the etching time for this sample was 1 second) the 2195 etches dark and the 6061 remains light. Note that the contrast in Fig. 6 is a material contrast while the contrast in Figs. 2 and 3 comes from different etch rates on the same 6061 material. Very considerable movement of the top 2195 can be seen into the bottom 6061 . The downward movement of 2195 in the central region of the vortex flow is balanced by an upward movement of 6061 near the outside of the rotational zone.

The cross section of Fig. 3 shows many features also shown in the plan view of Fig. 2. For example, it shows a region that etches dark on each side of the nib and corresponds to the rotational zone seen as an annulus in Fig. 2. A light gray area is seen on the retreating side (left) outside of the rotational zone. This region corresponds to the transition zone of Fig. 2 and is larger on the retreating side in both Figs. 2 and 3.

Regions devoid of material near the grooves are also seen in agreement with Fig. 2. The small bright white features in Fig. 3 are the copper tracer particles.

Notice that in Fig. 3 the flow in the top one third of the figure is dominated by the shoulder rather than by the threads of the nib and has a quite different flow pattern than material at the lower part of the nib. Flow in the lower two thirds of the cross section is of primary concern in this paper. Clockwise and counterclockwise welds (with the same tool) would have opposing vortex circulation directions and would be expected to have different interactions with the flow in the shoulder dominated region. This interaction is not yet understood, but it is believed that this interaction causes the differences between clockwise and counterclockwise welds. 
Returning to Fig. 2, it is further seen that dark arc shaped features intercalated with light material are left behind the weld. These features are seen coming off the rotational zone on the advancing rear side of the nib. Fig. 7 is a detail on the boundary between a dark and a light feature. In the dark region the grains are faceted and are the same size as those in the rotational zone. Also seen are rounded less heavily etched grains from the white intercalations seen in Fig. 2. Also seen in this figure is a particle of the copper tracer. Such tracer particles are found in various places in the dark arc shaped material behind the weld just as they were found in the rotational zone. The tracer is never found in the light material behind the weld.

Fig. 8 is an enlargement of the transition zone near arrow B in Fig. 2 from the retreating side of the nib. Grains elongated in the direction of rotation can be seen. These grains are parent metal subjected to entrainment by the rotating nib. If one assumes that the material on the retreating side of the nib is squeezed through this transition zone, there is a strain on the order of one half the nib diameter divided by the width of the transition zone or about $800 \%$.

An enlargement of this entrained material from the left hand side of the wake of the weld tool is shown in Fig. 9. Since copper tracer material is never seen in this light material, it is concluded that only material on the retreating side (that is to the left of the copper strip in Fig. 2) is entrained. On the other hand, material ahead but on the advancing side of the nib (and perhaps some from the retreating side near the center line also) is moved by entering onto the rotational zone, undergoing several revolutions, and finally dropping off in the wake of the nib. Microhardness tests of this entrained light etching material showed a Vicker's micro-hardness of 35 contrasted with a hardness in 
the dark bands of 95 and the reason for the differential etch (in spite of similar grain size) is apparent. The metal in the FSW weld zone thus consists of two streams of material with different histories and mechanical properties. The relative amounts of metal produced by these two processes will at least partially account for differing weld properties as weld schedule and nib are changed.

\section{CONCLUSIONS}

1. Material is moved around the nib in Friction Stir Welding by two processes:

a. Material on the advancing front side of a weld enters into a rotational zone that rotates and advances with the nib. This material is very highly deformed and sloughs off behind the nib in arc shaped features.

b. Material on the retreating front side of the nib is entrained (but never rotates around the nib) and fills in material on the retreating side of the nib wake.

2. Material transported by these two processes has very different thermo-mechanical histories and properties.

3. There is large vertical or vortex movement of material within the rotational zone caused by the wash and backwash of the threads. Material entering this zone thus follows an unwound helical trajectory formed by the rotational motion, the vortex flow, and the translational motion of the nib.

4. Material near the top of the weld (approximately the upper one third) moves under the influence of the shoulder rather than the threads on the nib. The interaction between the material moving in this upper zone and the lower thread dominated zone causes the 
differences between welds made with the same nib but in clockwise or counterclockwise directions. Flow in the upper shoulder dominated zone is under study.

\section{ACKNOWLEDGEMENTS}

The authors gratefully acknowledge the National Aeronautics and Space

Administration MURED program for support under Grant \# NAG8-1645. Likewise, the work never would have been completed without the insights and ingenuity of David Brown in the Department of Metallurgical and Materials Engineering 


\section{FIGURE CAPTIONS}

Fig. 1 Vortices seen in the weld zone of bimetallic weld of copper and aluminum. Light regions are copper and dark regions are aluminum. These vortices are roughly $40 \mu \mathrm{m}$ across, but scales vary greatly.

Fig. 2 Plan view at mid thickness of weld with a frozen nib. A copper foil marker was on the faying surface of the 6061 aluminum and is seen at top. A is a gap without material, $\mathrm{B}$ is the rotational zone or that rotates with the nib, and $\mathrm{C}$ is a transitional zone of material that is entrained by the rotating nib. Small bright particles in $B$ are tracer particles from the copper foil. The dark arc shaped bands behind the nib are discussed in the text.

Fig. 3 Cross section of a frozen nib. Bright copper particles can be seen throughout the dark rotational zone surrounding the nib. The transitional zone of Fig. 2 can be seen outside of the rotational zone and is larger on the retreating side than on the advancing side.

Fig. 4 Microstructure of rotational zone showing small angular grains with occasional copper tracer particles.

Fig. 5 Region ahead of nib showing copper foil on edge entering into rotational zone. Note the foil is immediately broken into small particles which appear at various places in the rotational zone.

Fig. 6 Weld made on a specimen with aluminum 2195 on top and 6061 aluminum below. The dark etching 2195 is carried downward by the vortex flow of the nib indicating large vertical movement of material during welding.

Fig. 7 Detail of arc shaped feature and region next to it showing both the dark and light material. Copper particles are seen in the dark material. Such particles are never seen in the light regions.

Fig. 8 Elongated grains at retreating side of Fig. 2 near arrow $C$. This material is being entrained by the rotating nib. 
Fig. 9 Enlarged picture of entrained grains (now recrystallized and equiaxed) behind weld on left side near D on Fig. 2. 
${ }^{1}$ W.M. Thomas, et al., 1991. Friction Stir Butt Welding. International Patent Application No. PCT/GB92102203 and Great Britain Patent Application No. 9125978.8.

${ }^{2}$ McClure, J.C., Feng, Z., Tang, W., Guo, X, "A Thermal Model of Friction Stir Welding", Proceedings $5^{\text {th }}$ Trends in Welding Research Conference, Stone Mountain, GA, June 1998, p. 590-595.

${ }^{3}$ Tang, W., Guo, X., McClure, J.C., Murr, L.E., Nunes, A., Heat Input and Temperature Distribution in Friction Stir Welding, Journal of Materials Processing and Manufacturing Science, Vol. 7, October 1998, p. 163-172.

${ }^{4}$ Murr, L. E., L. E. Murr, Ying Li, R. D. Flores, Elizabeth Trillo, and J. C. McClure, "Intercalation vortices and related microstructural features in the friction-stir welding of dissimilar metals," Materials Res. Innovations, 2(3), 150 (1998).

${ }^{5}$ Coligan, K., Material Flow Behavior during Friction Stir Welding of Aluminum, Welding Journal, July 1999, 229s-237s.

${ }^{6}$ Reynolds, A., Seidel, R.U., Simonsen, M."Visualization of Material Flow in an Autogeneous Friction Stir Weld", Proceedings $I^{\text {st }}$ International Symposium on Friction Stir Welding,

${ }^{7}$ L. E. Murr, E. A. Trillo, Y. Li, R. D. Flores, B. M. Nowak, and J. C. McClure, "SolidState Flow Associated with the Friction-Stir Welding of Dissimilar Metals," in Fluid Flow Phenomena in Metals Processing, N. El-Kaddah, D.G.C. Robertson, S. T. Johnson, and V. R. Voller (eds.), The Minerals, Metals and Materials Society, Warrendale, PA, 1999, p. 31.

${ }^{8}$ Ying Li, L. E. Murr, and J. C. McClure, "Flow Visualization and Residual Microstructures Associated with the Friction-Stir Welding of 2024 Aluminum to 6061 Aluminum," Materials Sci. \& Engng. A, 271, 213 (1999).

${ }^{9}$ L. E. Murr, C-S. Niou, E. P. Garcia, E. Ferreyra, J. M. Rivas, and J. C. Sanchez, "Comparison of Jetting-Related Microstructures Associated with Hypervelocity Impact Crater Formation in Copper Targets and Copper Shaped Charges," Mater. Sci. Engng., A 222, 118 (1997).

${ }^{10}$ L. E. Murr, E. P. Garcia, E. Ferreyra, C-S. Niou, J. M. Rivas, and S. A. Quinones, "Microtsructural Aspects of Hypervelocity Impact Cratering and Jetting in Copper," $J$. Mater. Sci., 31, 5915 (1996). 
${ }^{11}$ S. A. Quinones and L. E. Murr, "Correlations of Computed Simulations with Residual Hardness Mappings and Microstructural Observations of High Velocity and Hypervelocity Impact Craters in Copper," Phys. Stat. Sol. (a), 166, 763 (1998).

${ }^{12}$ S. A. Quinones and L. E. Murr, "Correlations of Computed Simulations with Residual Hardness Mappings and Microstructural Observations of High Velocity and Hypervelocity Impact Craters in Copper," Phys. Stat. Sol. (a), 166, 763 (1998).

${ }^{13}$ L. E. Murr, E. Ferreyra, S. Pappu, E. P. Garcia, J. C. Sanchez, W. Huang, J. M. Rivas, C. Kennedy, A. Ayala, and C-S. Niou, "Novel Deformation Processes and Microstructures Involving Ballistic Penetrator Formation and Hypervelocity Impact and Penetration Phenomena," Mater. Characterization, (Special Issue) 37, 245 (1996).

${ }^{14}$ C. Kennedy, R. Avalos, A. Bujanda and L. E. Murr, "Tungsten Heavy Alloy Ballistic Rod Penetration into Copper Targets: Microstructure Analysis and Computer Simulations," Chap. 63 in Fundamental Issues and Applications of Shock Wave and High-Strain-Rate Phenomena, K. P. Staudhammer, L. E. Murr, and M. A. Meyers (eds.), Elsevier Science, Amsterdam, 2001, p. 501.

${ }^{15}$ A.C. Nunes, Jr., E.L. Bernstein, and J.C. McClure, "A Rotating Plug Model for Friction Stir Welding" (Paper presented at the 81st American Welding Society Annual Convention, Chicago, Illinois, April 26-28, 2000).

${ }^{16}$ Guo, X., A Study of Friction Stir Welding on 6061-T6 Aluminum Alloy, M.S. Thesis, University of Texas at El Paso, El Paso, TX 79968, August, 1999.

${ }^{17}$ Coligan, op cit. 


\section{$1.941 \times+8$ basony}

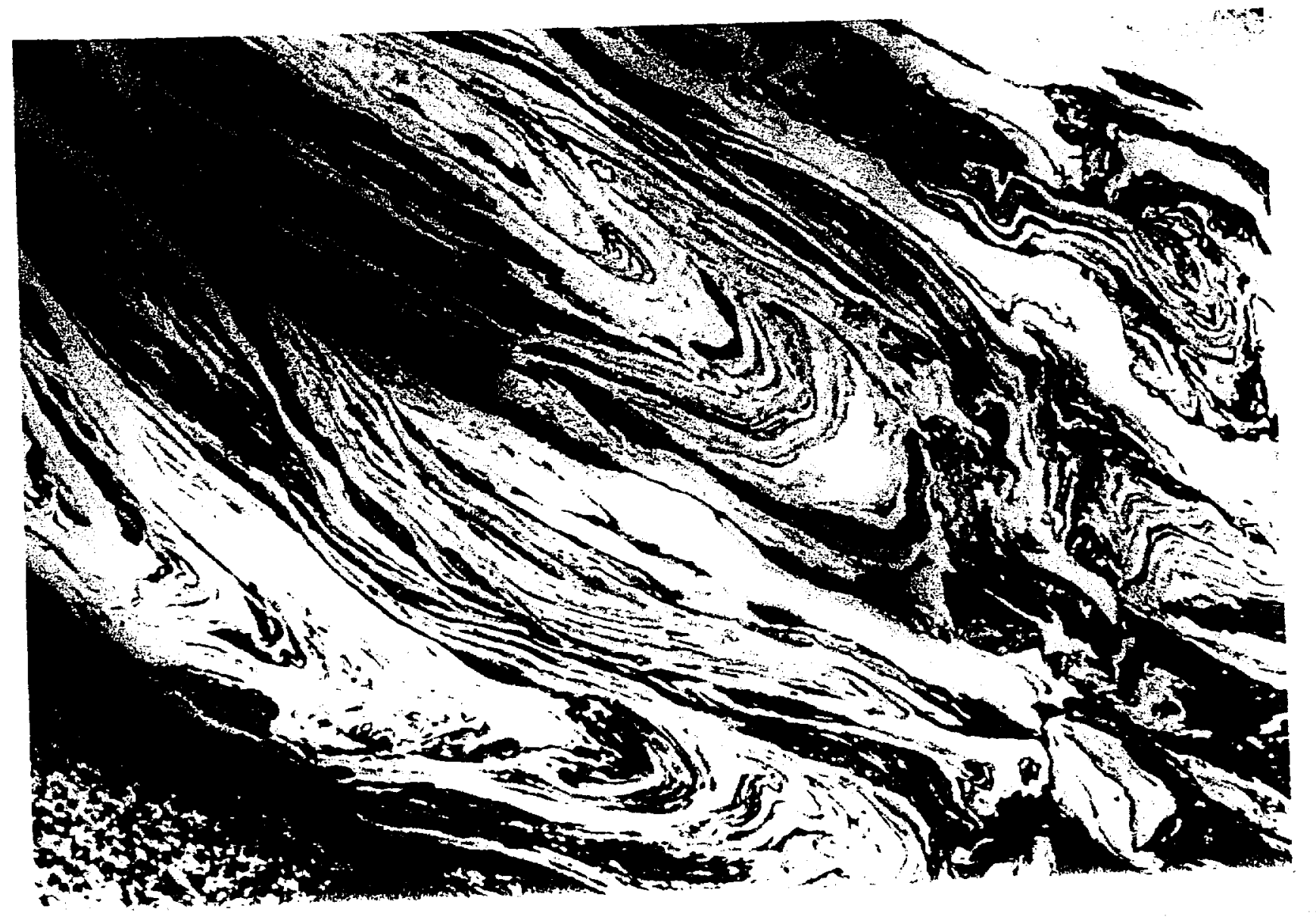



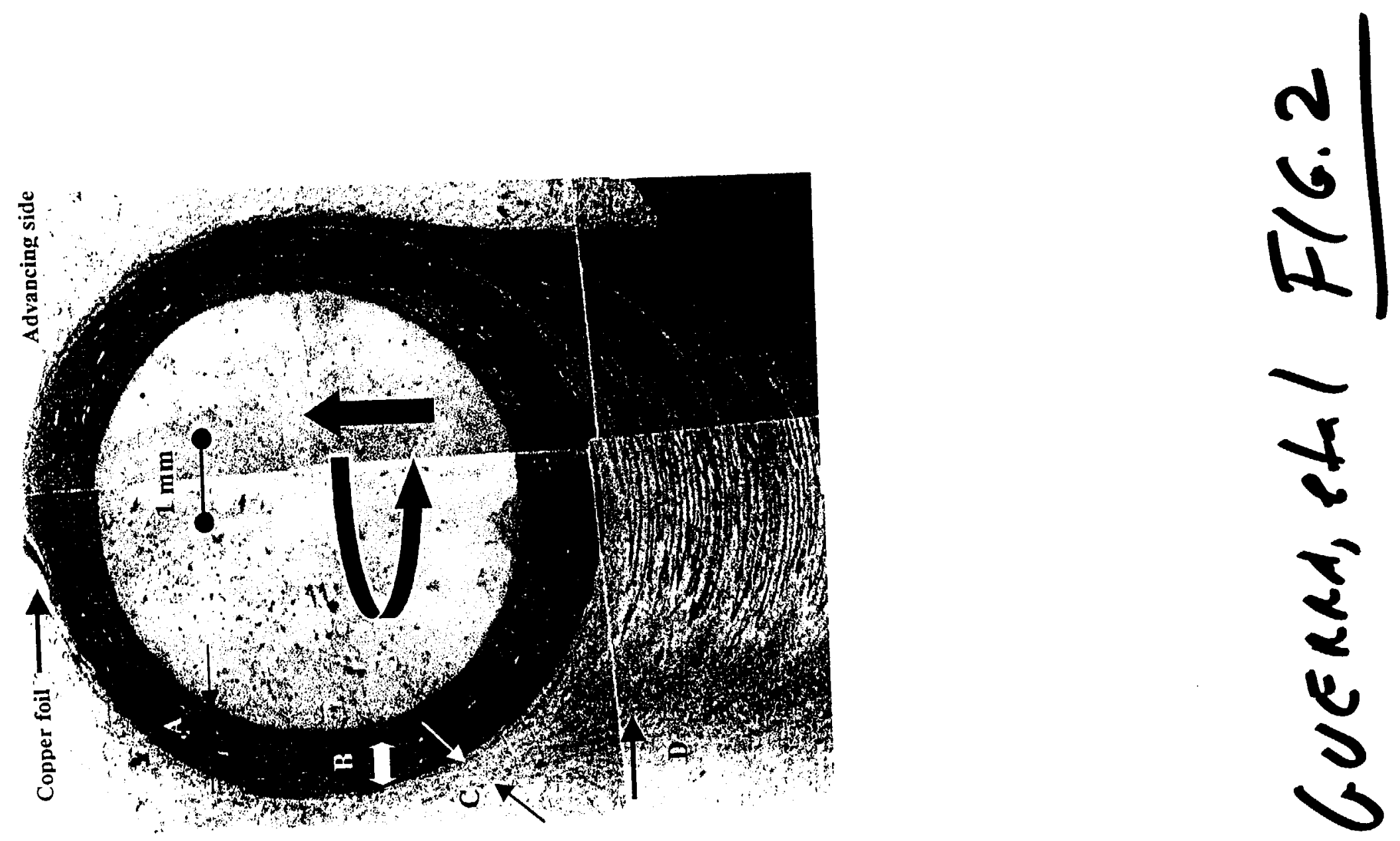


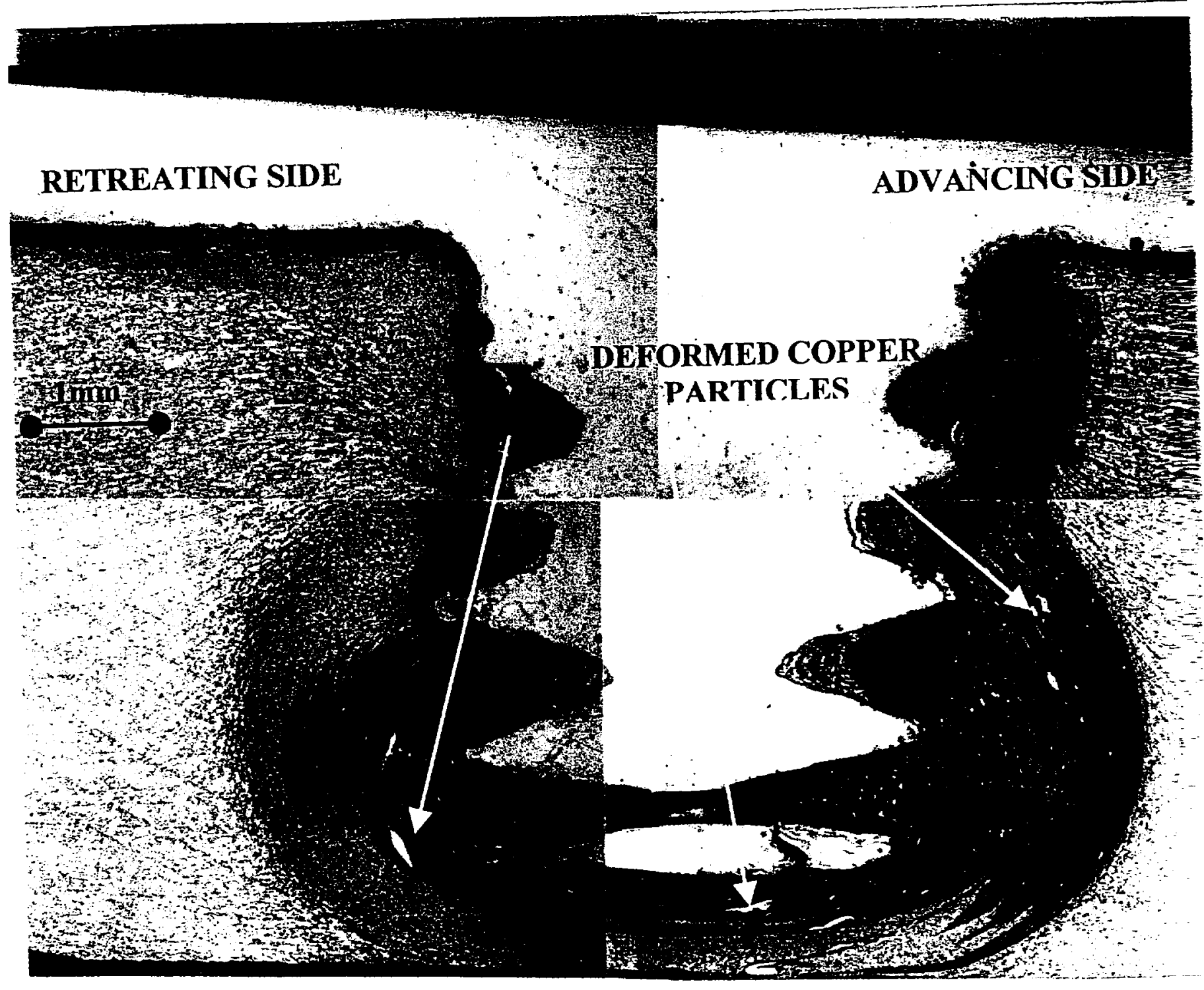

\section{Guexpes, at al Fig.3}




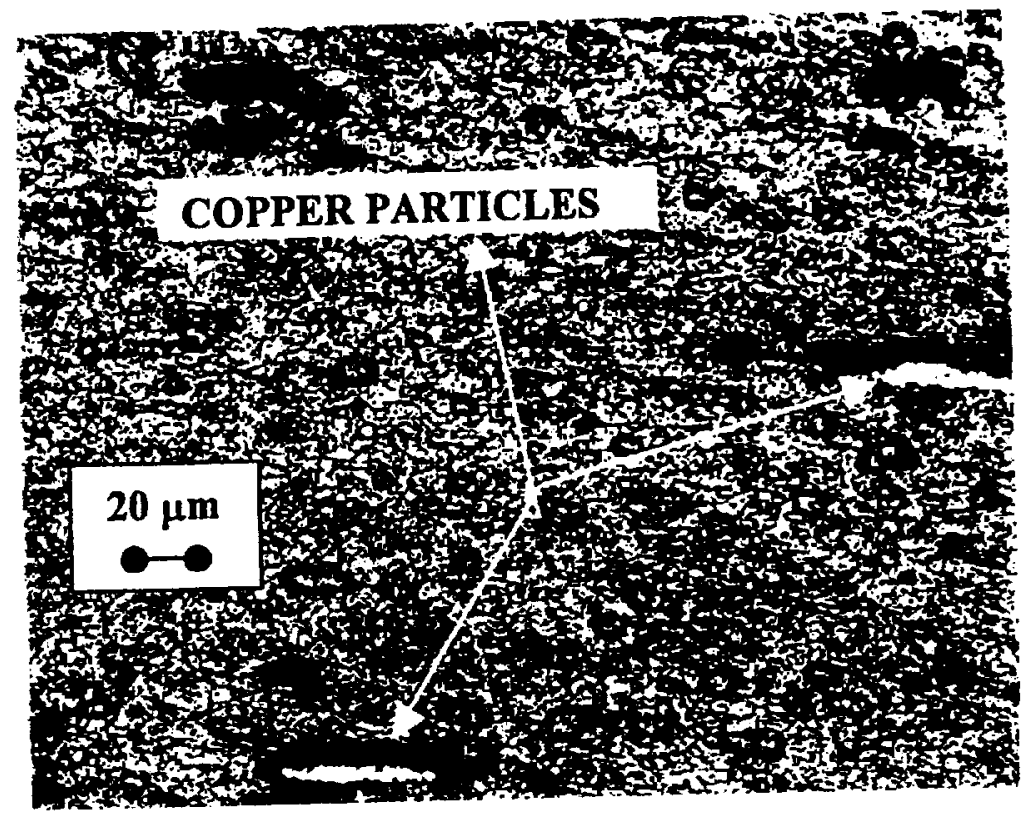

Gucesed, et al FIG.4 


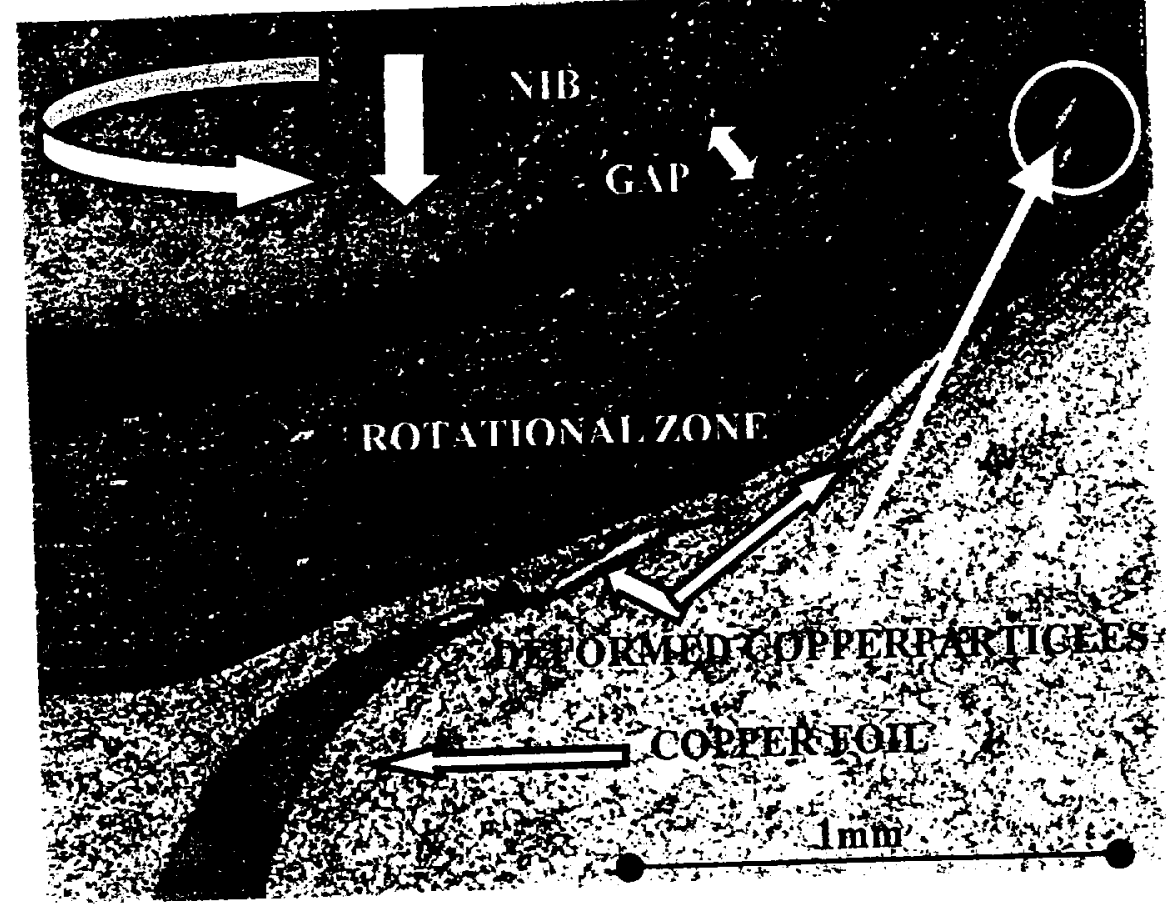

Guerar, et al FlG. 5 


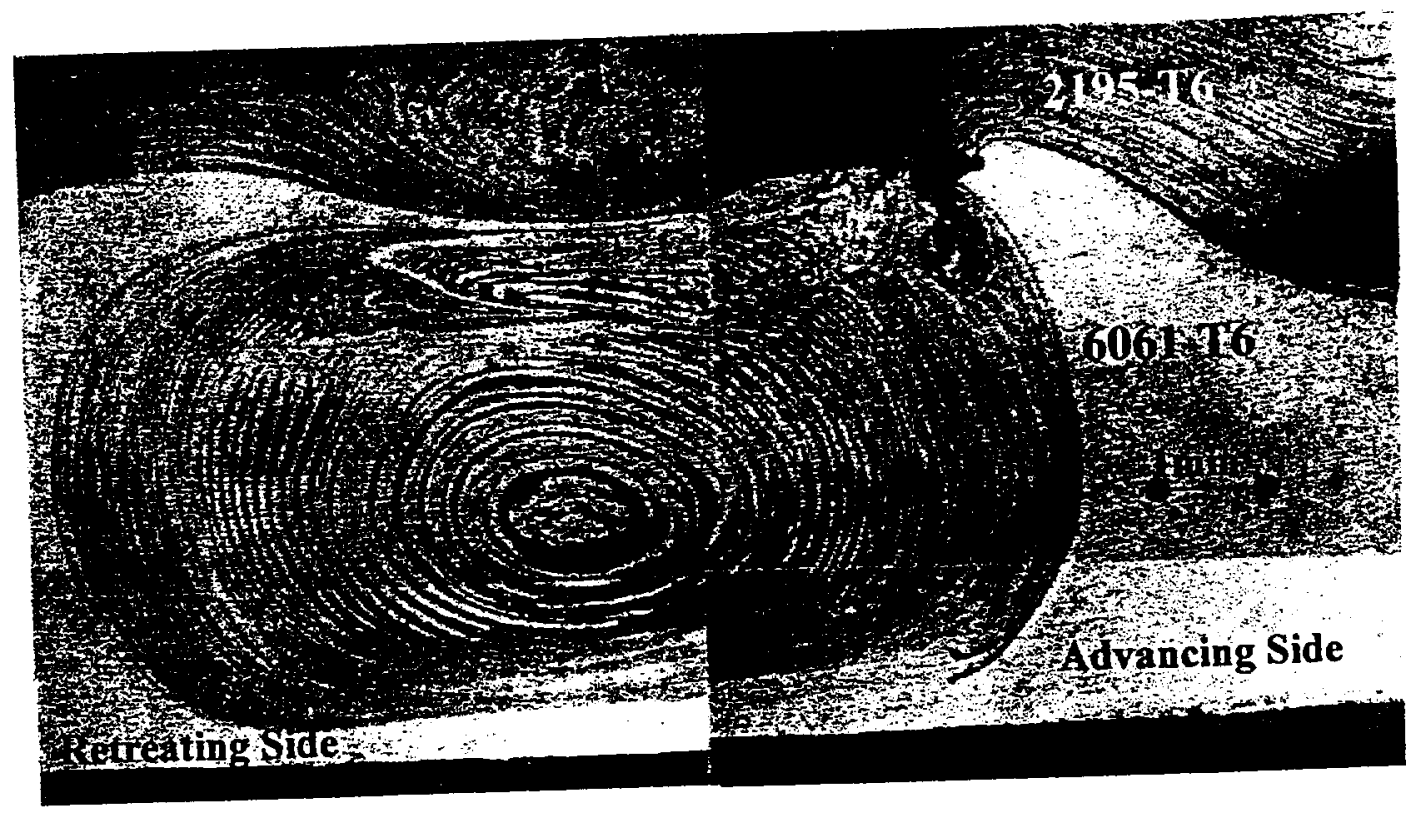

Gueran, etal Fíl. 


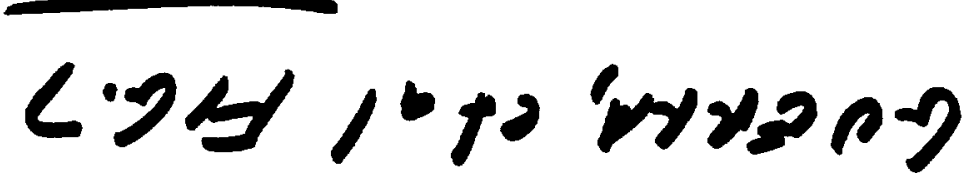

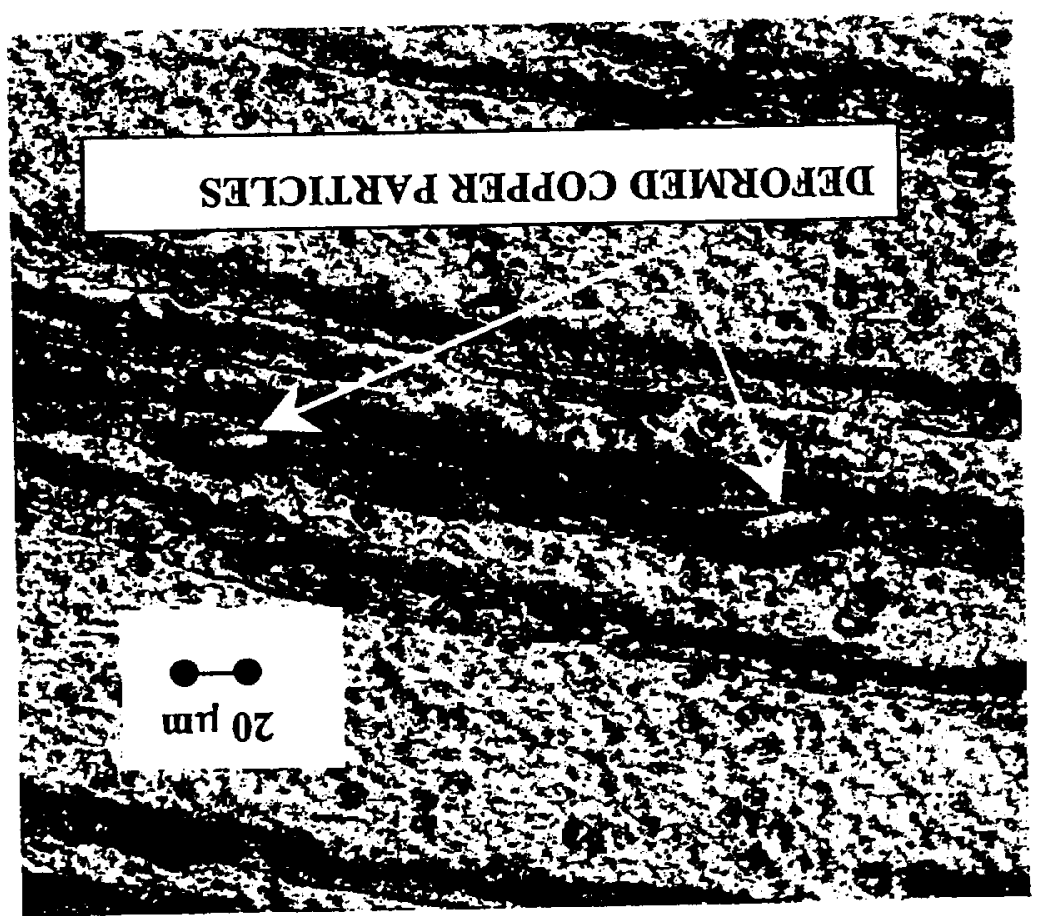




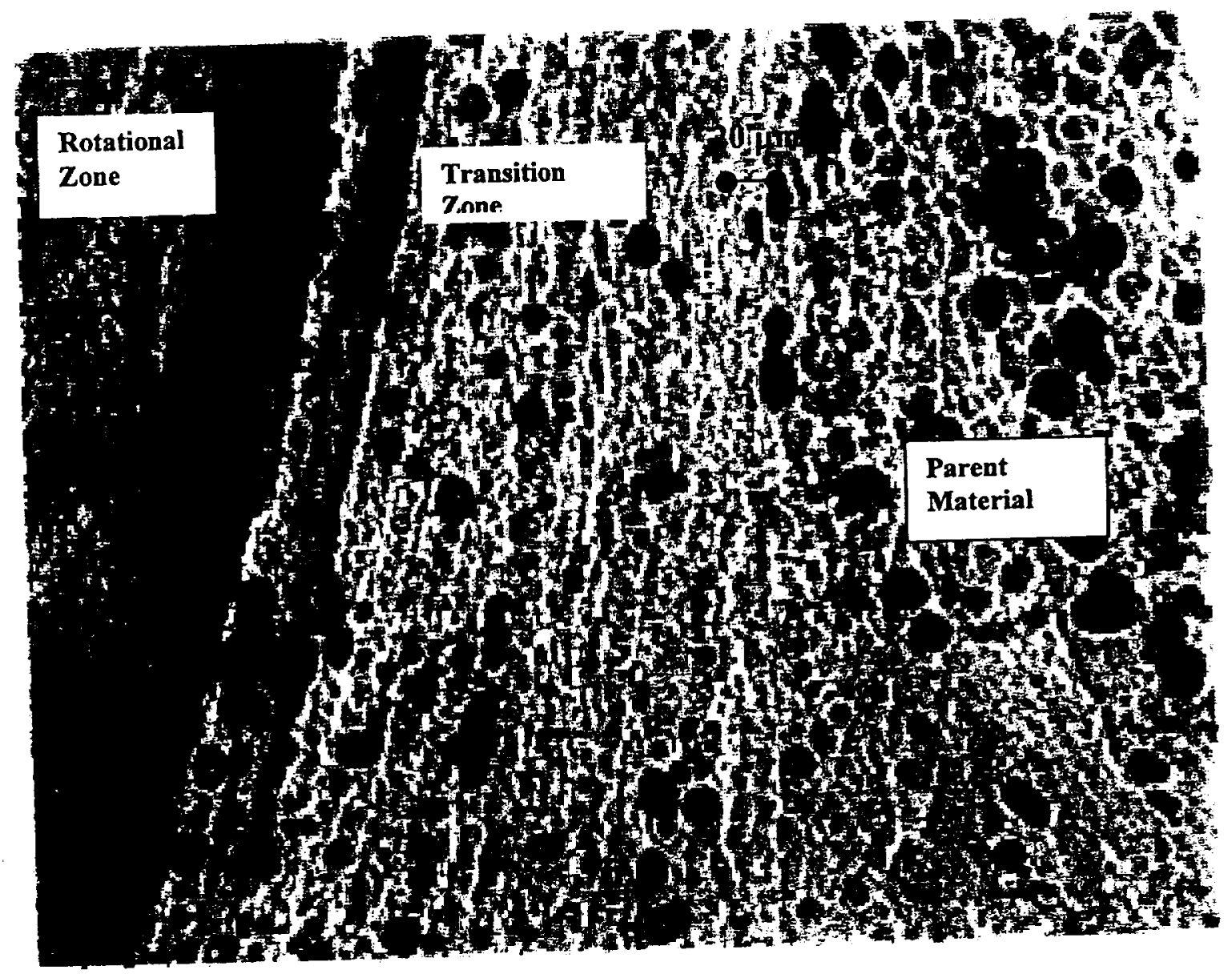

\section{Guenes, et al F/G. 8}



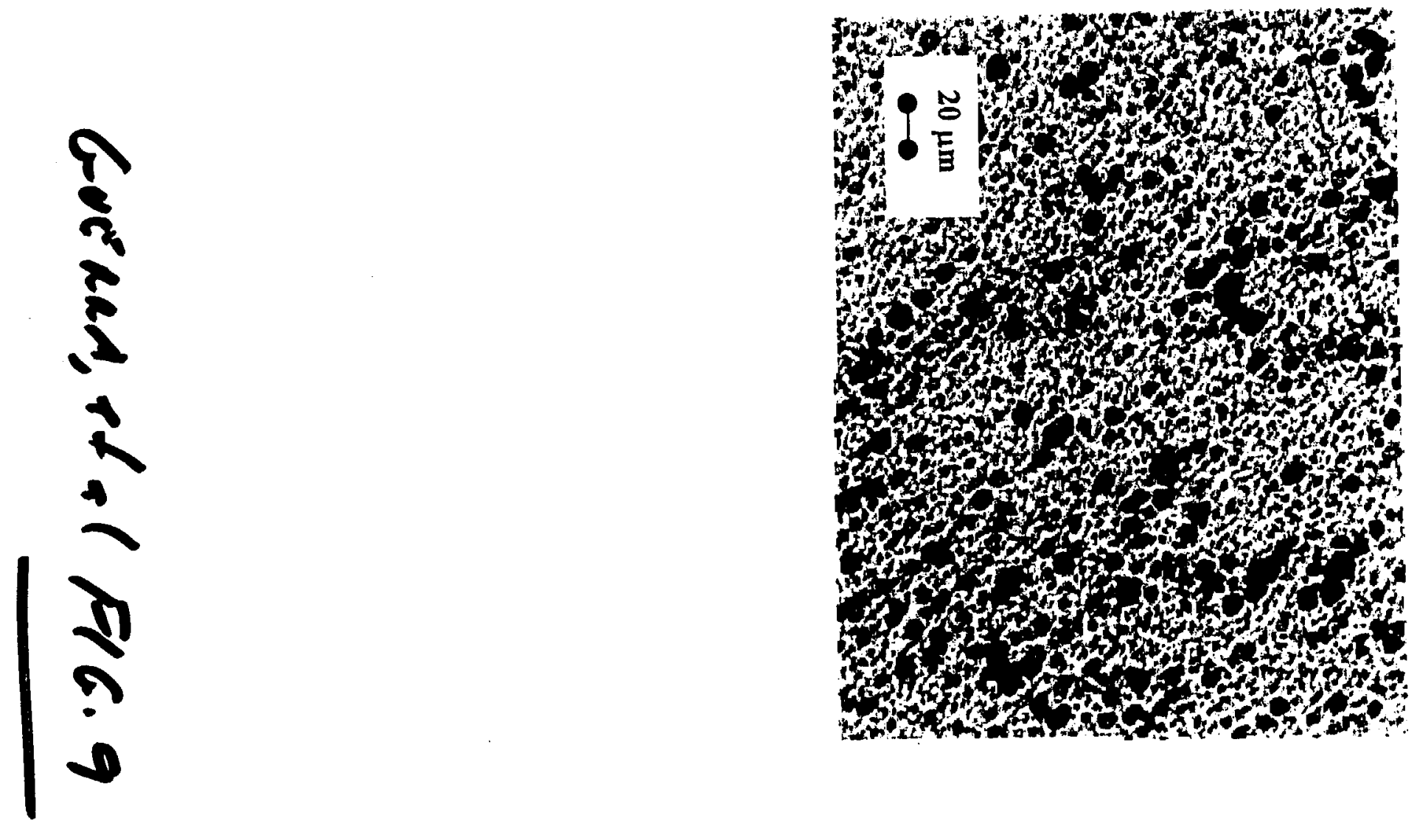